University of Nebraska - Lincoln

DigitalCommons@University of Nebraska - Lincoln

September 1989

\title{
Semiempirical determination of intermolecular anharmonicity in molecular crystals: The case of 1,2,4,5-tetrabromobenzene
}

\author{
R.C. Dye \\ University of Nebraska - Lincoln \\ Craig J. Eckhardt \\ University of Nebraska - Lincoln, ceckhardt1@unl.edu
}

Follow this and additional works at: https://digitalcommons.unl.edu/chemistryeckhardt

Part of the Chemistry Commons

Dye, R.C. and Eckhardt, Craig J., "Semiempirical determination of intermolecular anharmonicity in molecular crystals: The case of 1,2,4,5-tetrabromobenzene" (1989). Craig J. Eckhardt Publications. 20. https://digitalcommons.unl.edu/chemistryeckhardt/20

This Article is brought to you for free and open access by the Published Research - Department of Chemistry at DigitalCommons@University of Nebraska - Lincoln. It has been accepted for inclusion in Craig J. Eckhardt Publications by an authorized administrator of DigitalCommons@University of Nebraska - Lincoln. 


\title{
Semiempirical determination of intermolecular anharmonicity in molecular crystals: The case of 1,2,4,5-tetrabromobenzene
}

\author{
R. C. Dye a) and C. J. Eckhardt ${ }^{\text {b) }}$ \\ Department of Chemistry, University of Nebraska-Lincoln, Lincoln, Nebraska 68588-0304
}

(Received 23 December 1988; accepted 6 June 1989)

\begin{abstract}
The molecular crystal 1,2,4,5-tetrabromobenzene (TBB) has been studied using Brillouin scattering and lattice dynamical calculations. A method for mapping acoustic mode anharmonicity is developed that exhibits substantial directional behavior and correlates well with the directions of the molecular movements associated with the phase transition. It is shown that by comparing the calculated sound velocities with the experimental values, insight can be gained into the displacive phase transition and the lattice dynamics of TBB. The sound velocities are plotted for three crystallographic planes containing the crystal axes. The relationships between sound velocities and lattice dynamics are discussed. The phonon dispersion curves for three directions are also presented.
\end{abstract}

\section{INTRODUCTION}

Many properties of a material are determined by intermolecular forces. This is certainly the case for thermal and mechanical properties and partly true for electrical ones. Even in the case of a material's solubility, it can be argued that the extent of solvation is dependent upon how effectively one molecule can displace another. This, in turn, depends on intermolecular forces. To exploit the properties of a material to its fullest, a complete understanding of these forces is desirable. An approach to their study is in the crystal where the directional interactions are fixed. Also, much of the information acquired from studying simpler molecular systems can be extended to macromolecular systems. For these reasons, the study of molecular packing and intermolecular forces in the solid state has become an important area of research.

To determine how well intermolecular forces are understood, it becomes vital to compare theoretical predictions with experimental values. The accuracy of the theoretical calculations relies on the model used to make the predictions. Although simple models make more approximations, they are more general. A model that has shown a great deal of success in balancing the number of approximations and the accuracy of the values is the atom-atom summation scheme used with a fixed parameter set. ${ }^{1}$ The crystal's potential energy is usually obtained from this summation and permits calculation of molecular orientation, phonon frequencies, elastic properties, and lattice constants. An atom-atom potential model commonly used is the 6-exp Buckingham potential which provides a broad base for a general understanding of the potential energy. Proper parametrization gives the method the accuracy needed to compare the results to experimental values. This parametrization of the potential is important and methods of assessing its quality are desirable. In part, the significance of this study is to investigate such a method.

\footnotetext{
a) Work completed in partial fulfillment of requirements for the Ph.D. at the University of Nebraska-Lincoln.

${ }^{b)}$ Author to whom correspondence should be addressed.
}

A major consideration in lattice dynamical calculations is the incorporation of anharmonicity into the model. In the atom-atom potential model this can, in principle, be taken into account by expanding the lattice potential in powers of molecular displacements. However, for practical applications, this approach is unsuitable due to the complications involved. ${ }^{1}$ Only recently has a direct experimental measurement of the anharmonicity present in a lattice optical mode been made using piezomodulated Raman spectroscopy. ${ }^{2}$ With new techniques such as this, reasonable alternatives to line shape analysis for determining the anharmonicity in a lattice can be proposed. In this study a method is presented which semiempirically maps anharmonicity of acoustical modes.

Anharmonic acoustical phonons have been linked to structural phase transitions. ${ }^{3}$ They can also play a role in such things as superconductivity and conformational changes in biological molecules. ${ }^{1,3}$ Many of the bulk properties of a material are determined by the anharmonicity which is present. Knowing the extent of a phonon mode's anharmonicity enables phenomena such as structural phase transitions to be better understood. Therefore, the determination of the anharmonicity in a crystal is of material significance.

A further concern has been associated with the anisotropy of the potential. For example, the potential may behave harmonically in one direction but not another. The question then arises of whether this is best described by a different lattice dynamical formalism, such as the quasiharmonic approximation, or by adjustment of the model potential.

Recently, there has been theoretical and experimental interest in the lattice properties of 1,2,4,5-tetrabromobenzene (TBB) ${ }^{4,5}$ In this system, the lattice cohesion is mainly due to van der Waals interactions. This provides a large separation in energy between the internal molecular modes and the external lattice modes. Using this fact, theoretical calculations can be simplified by treating the molecules as rigid. Also, TBB is known to undergo a displacive structural phase transition thus implying that unusual acoustic phonon behavior exists. Therefore, TBB provides a good model for the study of anharmonicity. 
In this paper, a brief discussion of Brillouin scattering is presented. Subsequently, along with elastic constant values, the molecular structure and packing effects related to phonon velocities in TBB are treated in a following section, thereby providing a microscopic interpretation of the macroscopic measurements. Following that, an examination of the differences between the model potential and that of the lattice is presented. Finally, arguments for the phase transition mechanism in TBB are given.

\section{THEORY}

\section{A. Brillouin scattering}

In Brillouin spectroscopy the inelastic scattering of photons from acoustic lattice vibrations is observed. ${ }^{6}$ This process can be explained classically as Bragg diffraction from the moving grating formed by the vibrating periodic lattice. Acoustic phonons, which are the vibrations associated with translations of mass in the unit cell, move through the lattice at the speed of sound. This phonon velocity depends on how effectively the mass in the unit cell can be moved which, in turn, is directly related to the force constants holding the mass in place.

Scattering in a highly anisotropic medium admits fewer simplifying assumptions than generally made for higher symmetry lattices. The Brillouin equation for an anisotropic material is

$$
\delta v= \pm v_{0} v\left(n_{i}^{2}+n_{s}^{2}-2 n_{i} n_{s} \cos \theta\right)^{1 / 2} / c,
$$

where $\delta v$ is the Brillouin shift and $v_{0}$ is the frequency of the laser light. The velocity of the phonon is $v$ and $c$ is the velocity of light in vacuum. The indices of refraction of the incident and scattered light are $n_{i}$ and $n_{s}$, respectively, and $\theta$ is the scattering angle. A typical Brillouin spectrum is shown in Fig. 1.

From Eq. (1), it can be seen that the shift is dependent upon two different indices of refraction. In isotropic materials, Eq. (1) is reduced because the refractive indices are, for experimental purposes, identical. Thus for anisotropic materials, a precise determination of the Brillouin shift is substantially more complicated.

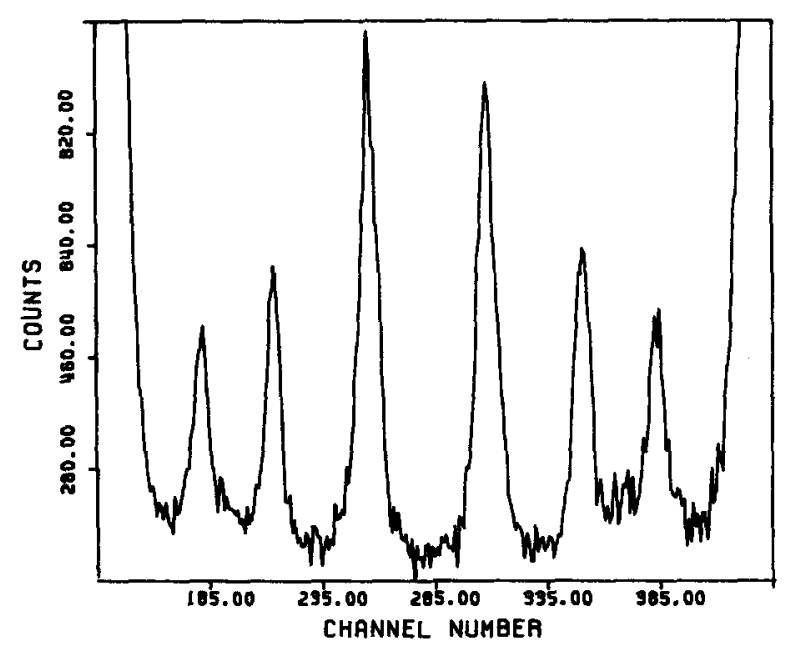

FIG. 1. TBB Brillouin spectrum with beam incident on the (110) face and light collected from the $(001)$ face.

\section{B. The harmonic approximation and anharmonicity}

The atom-atom potential method was used to determine the theoretical crystal energy. To utilize this, it is necessary to have a model potential which converges to a minimum value and closely represents the real system. For this, the atom-atom intermolecular Buckingham potential was employed,

$$
V_{i j}=-A_{i j} r_{i j}^{-6}+B_{i j} \exp \left(-C_{i j} r_{i j}\right)
$$

where $A_{i j}, B_{i j}$, and $C_{i j}$ are empirical constants whose values depend upon the type of atom-atom interaction between different molecular units. The values used for TBB are given in Table $\mathrm{I}$. The distance between the $i$ th and $j$ th atoms is given by $r_{i j} . V_{i j}$ is the crystal potential energy where summation is assumed. Since TBB has monoclinic symmetry, the energy can be taken as a seven-dimensional surface consisting of the $\mathbf{a}, \mathbf{b}$, and $\mathbf{c}$ lattice periods, the beta angle, and the three Euler angles which describe the orientation of the molecules in the unit cell. Under any cell deformations, the fractional positions of the molecules remain fixed. Therefore, to ensure that the crystal structure is in a strain-free equilibrium, the lattice energy and cell constants are minimized.

Once the crystal energy is determined, it becomes possible to calculate the lattice force constants from a given potential. ${ }^{7}$ A small displacement $\mathbf{u}_{i j}$ is considered about an equilibrium position $\mathbf{x}_{i j}$. This is expressed as

$$
\mathbf{r}_{i j}=\mathbf{x}_{i j}+\mathbf{u}_{i j} \text {. }
$$

Because the displacements are assumed to be small, $V_{i j}$ may be expanded in a Taylor series:

$$
V_{i j}=V_{i j}^{(0)}+V_{i j}^{(1)}+V_{i j}^{(2)}+V_{i j}^{(3)}+V_{i j}^{(4)}+\cdots,
$$

where

$$
V_{i j}^{(2)}=\frac{1}{2} \mathbf{k}_{i j}\left(\mathbf{u}_{i j}\right)^{2}
$$

with summation assumed $k_{i j}$ is the force constant. $V_{i j}^{(0)}$ is taken as the zero point energy. $V_{i j}^{(1)}$ expresses the force on the system and is zero at equilibrium. The third and higher order terms deal with the anharmonicity of the system and are not employed in the harmonic approximation. Therefore, even though the Buckingham potential is anharmonic, the determination of the force constants is made entirely within the harmonic limit since only the second order term in the expansion is used. This is justified because the constants are calculated only for small displacements from the bottom of the potential well. Examination of the potential in the region of these displacements reveals that it is harmonic. It is this harmonic shape that determines the force constants, not the overall shape of the entire Buckingham potential.

TABLE I. Contact parameters. ${ }^{\text {a }}$

\begin{tabular}{lrrr}
\hline \hline Interaction & $A$ & $B$ & $C$ \\
\hline $\mathrm{C} \cdots \mathrm{C}$ & & & \\
$\mathrm{C} \cdots \mathrm{Br}$ & 568 & 83680 & 3.60 \\
$\mathrm{C} \cdots \mathrm{H}$ & 730 & 78500 & 3.37 \\
$\mathrm{Br} \cdots \mathrm{Br}$ & 125 & 8766 & 3.67 \\
$\mathrm{Br} \cdots \mathrm{H}$ & 4580 & 149000 & 3.14 \\
$\mathrm{H} \cdots \mathrm{H}$ & 555 & 18050 & 3.44 \\
& 27.3 & 2654 & 3.74 \\
\hline
\end{tabular}

${ }^{\mathrm{a}}$ Empirical constants for Eq. (2) (Ref. 20). 


\section{EXPERIMENTAL}

\section{A. Sample preparation}

The beta phase of 1,2,4,5-tetrabromobenzene (TBB) packs in a monoclinic unit cell $\left(P 2_{1} / a\right)$ with two molecules located at centers of inversion. Lattice constants at $290 \mathrm{~K}$ are $\mathrm{a}=10.323 \AA, \mathrm{b}=10.705 \AA, \mathrm{c}=4.018 \AA$, and $\beta=102.37^{\circ} .8$

The starting material for the growth of TBB crystals was obtained from Aldrich Chemical with a reported purity of $98 \%$. TBB was purified by repeated vacuum sublimations yielding a snow-white material. Clear, colorless crystals, approximately $3 \times 5 \times 9 \mathrm{~mm}$ in size, were obtained by slow evaporation of a chloroform solution. The interiors of the crystals were of superior optical quality; however, some surfaces did have sporadic defects. Identification of the crystal faces by optical goniometry and morphological examination commonly showed well-developed (001), (110), (11̄0), ( $\overline{1} \overline{10})$, and ( $\overline{1} 10)$ faces. Interfacial angles were used in determining the scattering angle and position of the crystal.

\section{B. Instrumentation}

The Brillouin scattering instrumentation differs from that normally used and has been treated in greater detail elsewhere. ${ }^{9}$ This "four circle" system provides the versatility needed to obtain q-vector directions which are inaccessible by conventional means which require cutting the crystal. The source consists of a light-stabilized, single-moded, argon ion laser. Stabilization of the Fabry-Perot interferometer is achieved by use of a thermal box, a vibration-isolation table, and an active stabilization system. This system can maintain a finesse greater than 70 for an extended period of time. Conventional photon counting techniques and instrumentation are employed for the detection of the scattered light. For rapid data collection and display an IBM 9000 microcomputer is used.

\section{Method of measurement}

Three scattering schemes were employed: $90^{\circ}$, near $180^{\circ}$, and with incident and scattered beams normal to nonorthogonal, natural faces. Backreflection of the laser beam from the incident crystal surface provided an alignment point for the scattering instrumentation. Since this reflection is collinear with the vector normal to the face, the stage angle and crystal orientation can be set according to a predetermined scattering scheme with an accuracy of better than $0.5^{\circ}$ in the scattering angle.

In general, light normal to a crystal surface can propagate in the bulk in two directions. Because the incident beam is polarized and the scattered beam is analyzed, four scattering arrangements are measured. The axes of the optical indicatrix at $5145 \AA$ are $n_{x}=2.00, n_{y}=1.96$, and $n_{z}=1.63 .{ }^{10}$ Beam directions and directional refractive indices were obtained from interfacial angles and the indicatrix.

To locate superposed peaks, measurements were made with at least two free spectral ranges (FSR) extending from 0.898 to $1.420 \mathrm{~cm}^{-1}$. Crystalline quartz and liquid benzene were the calibration standards. ${ }^{1-13}$ The Brillouin shift was determined from the position of the peak center. ${ }^{14}$ Light de- polarization is often a problem ${ }^{15}$ and some depolarization of the incident and scattered beam was observed, e.g., the same energy shift in the scattering plane might be observed in the crossed polarizer arrangement. In this case, the resulting peak is attributed to breakthrough scattering. Causes for the depolarization include surface roughness and internal defects.

A complete experimental evaluation of the high temperature gamma phase of TBB has proven to be elusive. Two methods were attempted in trying to obtain a complete set of phonon velocities for this phase. First, crystals of TBB were grown above the phase transition and maintained at this temperature. These were very small and sublimed rapidly making them unsuitable for a complete determination of the phonon velocities. Next, an attempt was made to heat the beta phase crystals through the phase transition. However, the lattice shatters causing severe Rayleigh broadening. Phonon velocity measurements were taken within $0.5^{\circ} \mathrm{C}$ of the phase transition with no velocity shift of the phonons observed.

\section{CALCULATIONS}

\section{A. Experimental elastic constants}

The elastic constant tensor for a monoclinic crystal consists of 13 independent coefficients. The computation of the elastic constants requires solution of the Christoffel equation for different directions in the lattice ${ }^{16}$ :

$$
\left|\Gamma_{i j}(\mathbf{q})-\rho v^{2} \mathbf{l}\right|=0
$$

in which $\rho$ is the crystal density, $v$ is the elastic wave velocity, and $\Gamma_{i j}(\mathbf{q})=c_{i k j l} q_{k} q_{l}$. The elastic constants $c_{i k j l}$ are in full index notation; $q_{k}$ and $q_{l}$ are the direction cosines for the angle of the wave vector to the orthogonal reference axes $k$ and $l$. When the elastic constants are known, the solution of the Christoffel equation is trivial. However, it is the elastic wave velocities which are determined by experiment. Generally, the elastic constants are solved by selecting special wave vector directions that have simple elastic constant dependence. For example, the $(010)$ longitudinal wave for a monoclinic crystal is determined only by the $c_{22}$ element. This approach, however, is very limited since only a very few propagation directions with single elastic constant dependence are possible.

Cutting and polishing the crystal is usually resorted to in order to obtain special propagation directions that do not form naturally. This can severely degrade the surface as well as generate undetected cracks and other defects in the bulk. This approach also requires large crystals which are more prone to high defect concentrations which seriously affect the Brillouin line shapes and their intensities, and increases the Rayleigh wings.

By solving for the special directions first, the errors in the wave velocities accumulate and propagate so that the last determined tensor elements are associated with large uncertainties. Ambiguities also arise when solving the coupled quadratic or cubic equations that express the velocity dependence of the elastic constants.

In this study a different approach based on a linear leastsquares procedure is used. The method was first applied to 
cubic crystals ${ }^{17}$ and has recently been extended to lower symmetry crystals. ${ }^{18,19}$

The procedure consists of an iterative optimization algorithm which starts with an arbitrary set of elastic constants. Each iteration solves the Christoffel determinants for their eigenvalues $\rho v_{\text {calc }}^{2}$. Next, the program calculates an error vector $\mathbf{e}$ between the calculated eigenvalues and the observed eigenvalues $\rho v_{\mathrm{obs}}^{2}$ obtained from experiment. The square of the error vector

$$
\mathbf{e}^{T} \mathbf{e}=\Sigma\left|\rho v_{\mathrm{obs}(q, j)}^{2}-\rho v_{\mathrm{calc}(q, j, C)}^{2}\right|^{2}
$$

is minimized by systematically varying the elastic constants. The iterations continue until there is no further improvement in the values. Appropriate weights are added to reflect the uncertainty in the observed measurements. Increasing the number of observed phonon directions improves the results rather than cause greater ambiguities as in sequential calculations. This method should, therefore, provide a better overall scheme for the determination of the complete set of elastic constants. Furthermore, it does not rely on special propagation directions, and thus the errors do not accumulate and the ambiguities arising from solving quadratic or cubic equations are eliminated. This approach also removes subjective judgments such as neglecting certain elastic constants which are employed in subsequent calculations. And finally, it eliminates the experimental difficulties associated with cutting and polishing the crystal.

\section{B. Lattice dynamical calculation of elastic constants}

In this study, a set of elastic constants obtained from theoretical calculations are also presented. The method is described elsewhere in greater detail. ${ }^{18}$ The first lattice dynamical calculation of TBB was reported by Burgos and Bonadeo. ${ }^{20}$ The results of their calculation do not agree well with the experimentally determined Raman active lattice modes. ${ }^{4} \mathrm{~A}$ likely reason for this discrepancy is that the crystal energy was not minimized with respect to the unit cell constants. This results in values for a strained, nonequilibrium lattice. Therefore, another calculation has been performed with the necessary minimization. ${ }^{4}$ However, for the determination of theoretical elastic constants, the lattice dynamical determinations for the acoustic mode frequencies have to be evaluated at several q values. In the present study 15 different q-vector directions were used to determine the theoretical acoustic mode frequencies by employing force constants determined within the harmonic limit of the potential. Once the frequencies are ascertained, the theoretical elastic constants are calculated in the same manner as the experimental values.

\section{RESULTS AND DISCUSSION}

The results of the elastic constants' determination are checked for accuracy in several ways. First, a direct comparison is made between the measured velocities and those calculated from the best set of elastic constant values. In this work, the average error between these values was $3.0 \%$ for the theoretical set and $5.0 \%$ for the experimental set. The function of Eq. (7) is expressed such that its value should be a minimum. Therefore, the lower the function value, the bet- ter the elastic constant values should be. In order for the elastic constant set to be teal, the determinant of the $6 \times 6$ matrix must be positive. This condition was met.

The results of the elastic constant determinations are given in Table II. To clarify the role packing and molecular structure have on phonon propagation, the phonon velocity projections are plotted onto the three crystal planes. The top halves of Figs. 2, 3, and 4 show the experimental velocities and the bottom halves display the calculated velocities. The projections of the TBB molecules onto their respective planes are also given.

The comparison of experimental to theoretical values is of interest because it shows how well the system is modeled by the harmonic part of the potential. A major cause for differences is that the experimental system is dynamic, whereas the calculated system is assumed to be static. The measure of dynamic action can be related to the amount of anharmonicity in the lattice. ${ }^{1}$ For a given energy content, a harmonic system will not exhibit as much motion as one that is anharmonic. For real solids, some directions can be harmonic while others are very anharmonic and this is manifested by the varying sound velocities in different directions in the crystal. The difference of the theoretical and the measured sound velocities provides a measure of the anisotropy of the anharmonicity of the acoustic modes.

Because the model potential describes essentially harmonic modes, and the experimental modes may be regarded as being harmonic with anharmonic contributions [see Eq. (4) ], the difference between the theoretical and experimental sound velocities should provide a description of the anharmonicity in the system relative to the theoretical potential model used in the study. Alternatively, the difference between the modes may be due to an insufficient parametrization of the model potential. It is then of interest to establish how the model potential is inadequate. However, if the theoretical potential closely models the harmonic part of the real system, then information about the real system can be acquired. Hereinafter, "harmonic" and "anharmonic" will refer to these aspects of the theoretical potential model.

The longitudinal mode velocities in all three planes containing the crystal axes are predicted by theory to be within $5 \%$ of the experimental values. This implies that these inter-

TABLE II. TBB elastic constants. $\left(10^{9} \mathrm{dyn} / \mathrm{cm}^{2}\right.$.)

\begin{tabular}{lrrr}
\hline & Calc. & Expt. & Diff. \\
\hline C11 & 138.1 & 142.5 & 4.4 \\
C12 & 118.4 & 125.8 & 7.4 \\
C13 & 67.6 & 52.4 & 15.2 \\
C15 & 49.9 & 49.4 & 0.5 \\
C22 & 154.4 & 138.0 & 16.4 \\
C23 & 62.8 & 31.7 & 31.1 \\
C25 & 58.2 & 61.8 & 3.6 \\
C33 & 150.7 & 139.3 & 11.4 \\
C35 & -0.8 & -2.6 & 1.8 \\
C44 & 45.9 & 65.4 & 19.5 \\
C46 & 67.5 & 58.7 & 8.8 \\
C55 & 55.6 & 49.8 & 5.8 \\
C66 & 134.4 & 105.1 & 29.3 \\
\hline \hline
\end{tabular}




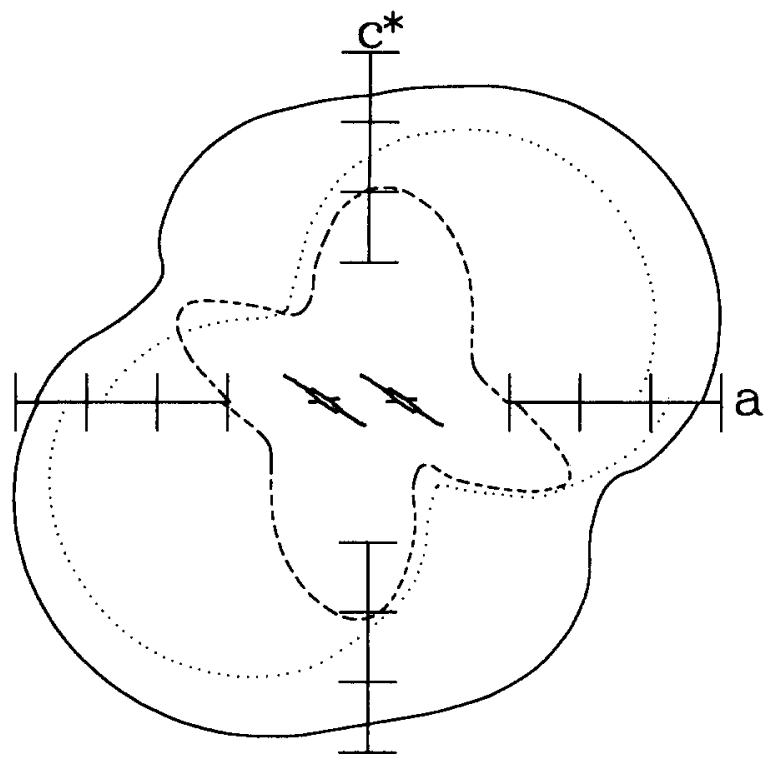

FIG. 2. Sound velocity diagram (ac* plane): experimental (top), calculated values (bottom). One division equals $0.5 \mathrm{~km} / \mathrm{s}$. Modes: quasilongitudinal (solid); quasitransverse (dotted); and quasitransverse out-of-plane (dashed). The interior shows the corresponding projection of the TBB unit cell packing.

actions are well described by the harmonic approximation. On the other hand, some transverse mode velocities deviate from theory by more than $20 \%$. To demonstrate this, Figs. 5 , 6 , and 7 show the amount and direction of deviation between theoretical and experimental mode velocities.

The transverse mode velocities in the plane generally show a $15 \%$ difference between theoretical and experimental values. One exception to this is in the $a b$ plane where a difference of $22 \%$ is observed in four areas. The large difference in values in these directions suggests that the develop-

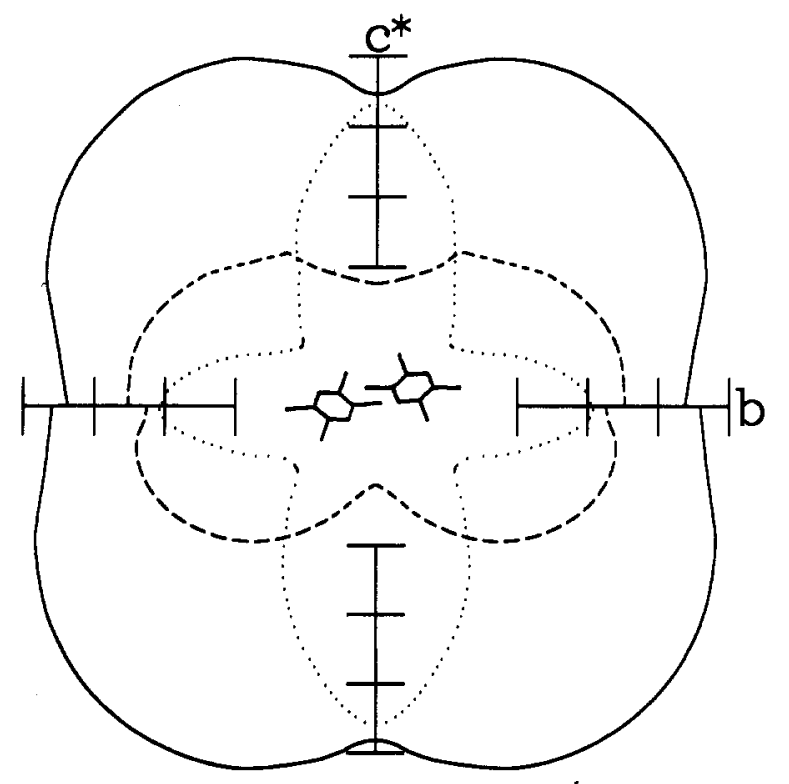

FIG. 3. Sound velocity diagram (bc* plane): experimental (top), calculated values (bottom). One division equals $0.5 \mathrm{~km} / \mathrm{s}$. Line identifications are as in Fig. 2. The interior shows the corresponding projection of the TBB unit cell packing.

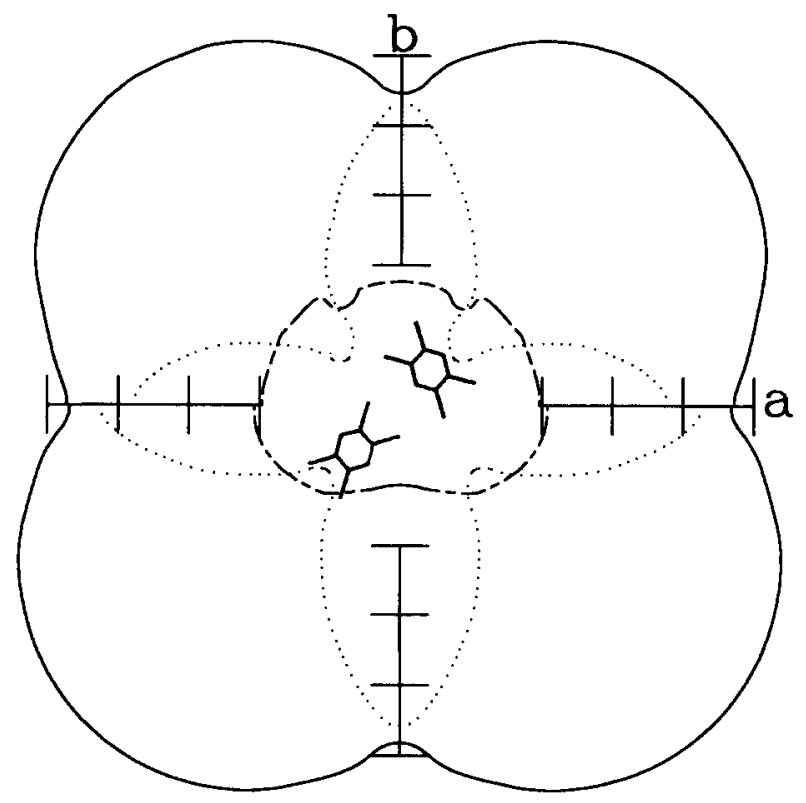

FIG. 4. Sound velocity diagram (ab plane): experimental (top), calculated values (bottom). One division equals $0.5 \mathrm{~km} / \mathrm{s}$. Line identifications are as in Fig. 2. The interior shows the corresponding projection of the TBB unit cell packing.

ment of the lattice dynamics in the harmonic approximation fails to provide an adequate description of the lattice potential. Such large deviations imply that significant anharmonicity is likely to be associated with these modes in the real lattice. These four areas may be correlated with the direction of the TBB molecular movement in the phase transition (Fig. 8).21

The transverse mode velocities out of the plane show the greatest deviation from theory. It is apparent from Figs. 6 and 7 , that this out-of-plane motion of the molecule may

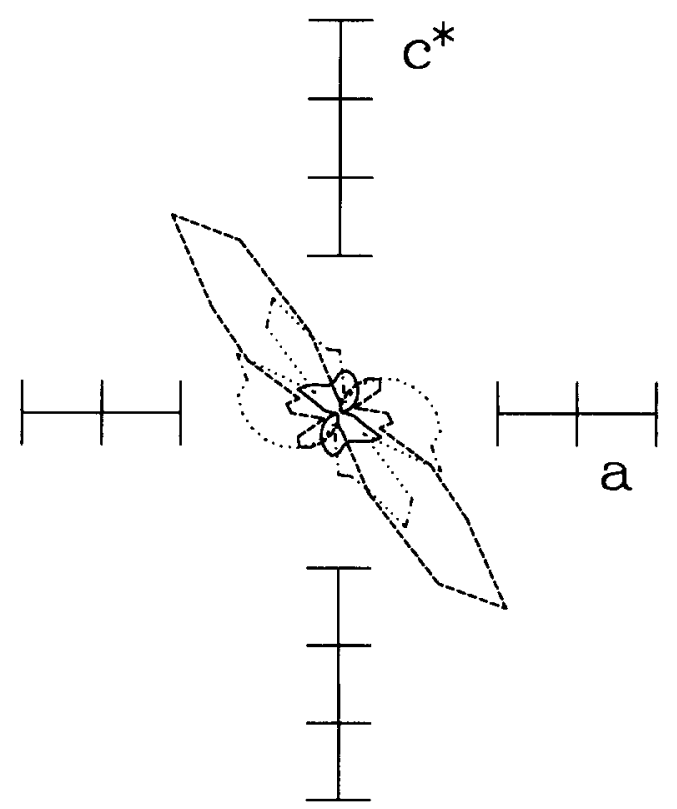

FIG. 5. Anharmonicity diagram (ac* plane). Scale is $5 \%$ relative difference. Modes: quasilongitudinal (solid); quasitransverse (dotted); and quasitransverse out-of-plane (dashed). 


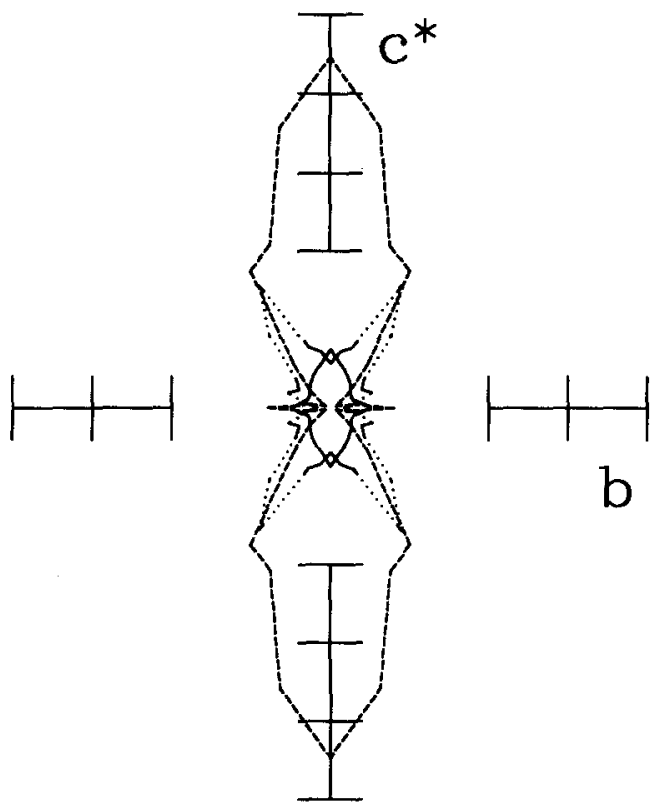

FIG. 6. Anharmonicity diagram (bc* plane). Scale is $5 \%$ relative difference. Modes: quasilongitudinal (solid); quasitransverse (dotted); and quasitransverse out-of-plane (dashed).

agree rather well with the Buckingham potential in some directions, such as the a direction in the $a b$, or the a direction in the $a c$ planes. However, this is not so for the $b$ direction of the $a b$ plane and the $\mathrm{c}^{*}$ direction in the $b c$ plane which show substantial deviations that can be associated with significant bromine-bromine interactions. The projection of the molecule in the $a b$ plane demonstrates that major bromine-bromine interactions occur in the b direction. Again, in the $b c$ plane a large bromine-bromine interaction is observed along the $c^{*}$ direction. This consistent observation is quite sugges-

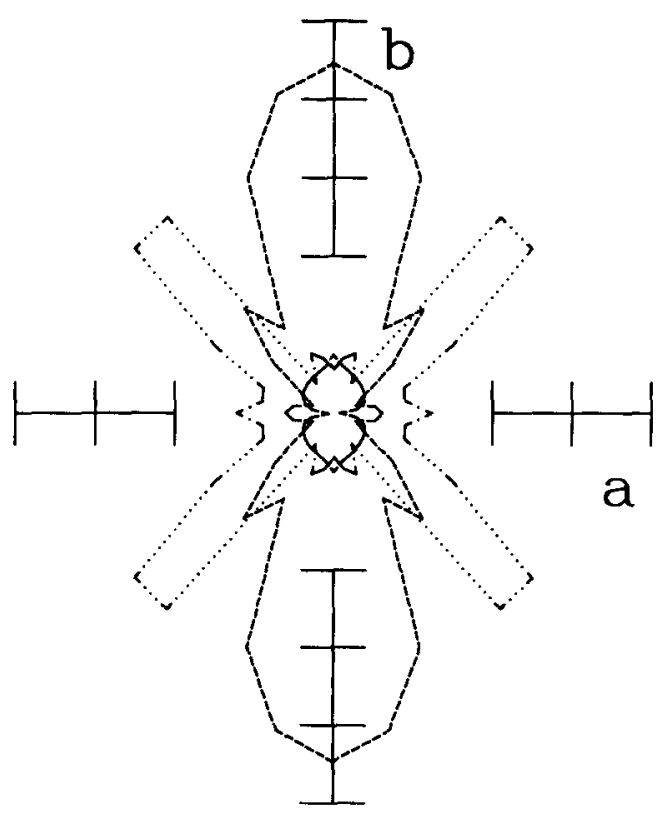

FIG. 7. Anharmonicity diagram (ab plane). Scale is $5 \%$ relative difference. Modes: quasilongitudinal (solid); quasitransverse (dotted); and quasitransverse out-of-plane (dashed).

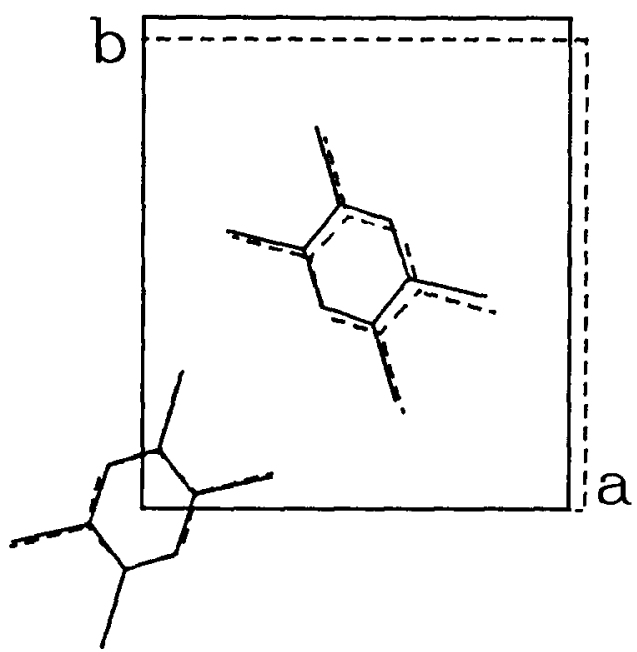

FIG. 8. Overlaid views of the unit cells of the beta (dashed line) and gamma (solid line) phases of TBB viewed down the $\mathrm{c}^{*}$ axis.

tive that this type of interaction does not follow the harmonic potential and lacks proper characterization. However, the bromine-bromine, end-to-end, interactions show reasonable agreement between theory and experiment. An improvement in understanding these interactions may be achieved by having directionally dependent potentials.

From the phonon velocity plots, it thus becomes possible to gain insight into the structural phase transition of TBB. When the lattice undergoes the phase change, the molecules will move in the most favored direction. The theoretical and experimental values suggest the same path of least resistance. A projection of this path is best observed in the $a b$ plane (Fig. 4). The transverse velocity in the plane exhibits a very low velocity at about $45^{\circ}$ off axis. Since the force constant is directly related to the square of the velocity, movement in this direction would be favored. The correlation between the movement of the molecules and the slow phonon modes becomes apparent when Figs. 4 and 8 are compared. From this, and observing that the same directions deviate strongly from the harmonic approximation, a mechanism for the phase transition in TBB is therefore inferred. This suggests that any movement of the TBB molecule during the phase transition occurs mainly in the $a b$ plane. The interactions allowing the transition seem to lie approximately $45^{\circ}$ off the a axis in the $a b$ plane. If the interactions in these regions were stronger, or followed a largely harmonic potential, the displacive phase transition might not occur.

The experimental and theoretical elastic constant values are compared in Table II. The agreement between values is relatively good; both sets have the same sign and agree within an order of magnitude. In other elastic constant studies where theoretical values are compared, this is not always the case $^{22}$ because of accumulated error. The present method of minimizing the values eliminates this and permits other considerations for discrepancies.

Even though only a small portion of the dispersion curve is used in determining the elastic constants, it is rather informative to examine the entire curve. Therefore, theoretical curves were calculated and are shown in Fig. 9. The 
[00६]

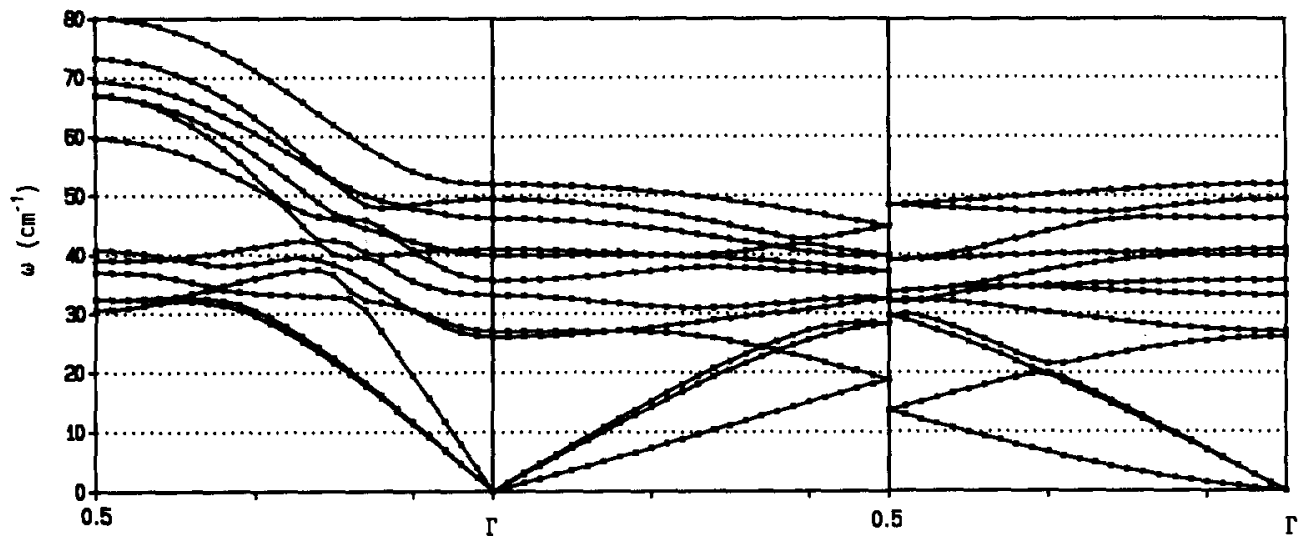

FIG. 9. Calculated phonon dispersion curves for TBB. Evaluated from reduced wave vector $q=0$ to Brillouin zone boundary in $\mathbf{a}^{*}, \mathbf{b}^{*}$, and $\mathbf{c}^{*}$ directions. slopes of the curves indicate the extent of mode localization. A large slope implies that the mode is delocalized and a small slope indicates a very local mode. The large amount of delocalization shown in the curves appears to be associated with the bromines and may be due to their significant polarizability. Since the lattice dynamics of halogenated hydrocarbons are not well characterized, nor extensively studied, further investigation is indicated. Of course, neutron scattering experiments would be most informative. Generally, before neutron scattering experiments are conducted to measure the dispersion curves, theoretical curves are needed for proper execution of the experiment. Those presented here should prove useful for such studies.

\section{CONCLUSION}

This work reports the sound velocities in numerous directions of crystalline TBB measured by Brillouin scattering. From these, a complete set of elastic constants are calculated. Also, by using the Buckingham potential, a full set of theoretical elastic constants and phonon velocities are obtained and compared. Straightforward arguments give some insight into the relation of the phonon propagation to the molecular packing.

Two directions of large lattice anharmonicity are expected: the direction of significant bromine-bromine interactions and the direction of the molecular movement in the phase transition. In both cases large differences between the velocities of the theoretical harmonic modes and the experimental modes were encountered.

Further, the discrepancies between the measured and calculated velocities indicate that explicit consideration of anharmonicity is warranted. For instance, the intermolecular interaction of end-to-end bromines may be quite different from bromines that are above and below one another, even though their interatomic distances may be the same. This may be expected to lead to further refinement of theoretical lattice dynamical calculations.

With more studies such as this, it may become possible to predict whether or not a material will undergo a phase transition and, if so, what the nature of the transition may be. Determining the details of the molecular movement during a phase transition may even be possible. Finally, it should provide an additional and direct measure of the quality of a model potential.

\section{ACKNOWLEDGMENT}

Support for this research by a grant from 3M Corporation is greatly appreciated.

'A. I. Kitaigorodsky, Molecular Crystals and Molecules (Academic, New York, 1973).

${ }^{2}$ K. M. White and C. J. Eckhardt, Phys. Rev. Lett. 59, 574 (1987).

${ }^{3}$ H. Z. Cummins and A. P. Levanyuk, Light Scattering Near Phase Transitions (North-Holland, Amsterdam, 1983).

${ }^{4}$ K. M. White and C. J. Eckhardt, J. Chem. Phys.90, 4709 (1989).

${ }^{5}$ E. Burgos and H. Bonadeo, Chem. Phys. Lett. 57, 125 (1978).

${ }^{6} \mathrm{~W}$. Hayes and R. Loudon, Scattering Of Light By Crystals (Wiley, New York, 1978).

${ }^{7}$ A. J. Pertsin and A. I. Kitaigorodsky, The Atom-Atom Potential Method. Applications to Organic Molecular Solids (Springer, Berlin, 1987).

${ }^{8} \mathrm{G}$. Gafner and F. H. Herbstein, Acta Crystallogr. 13, 706 (1960).

${ }^{9}$ R. C. Dye, J. F. Sartwell, and C. J. Eckhardt, Rev. Sci. Instrum. (in press).

${ }^{10}$ K. M. White, Ph.D. thesis, University of Nebraska, 1987.

'I. L. Fabelinskii, Molecular Scattering Of Light (Plenum, New York, 1968).

${ }^{12}$ S. M. Shapiro, R. W. Gammon, and H. Z. Cummins, Appl. Phys. Lett. 9, 157 (1966).

${ }^{13}$ H. Z. Cummins and R. W. Gammon, Appl. Phys. Lett. 6, 171 (1965).

${ }^{14}$ H. Z. Cummins and A. P. Levanyuk, in Ref. 3.

${ }^{15}$ M. C. Tobin, Laser Raman Spectroscopy (Wiley, New York, 1971).

${ }^{16} \mathrm{G}$. Venkataraman, L. A. Feldkamp, and V. C. Sahni, Dynamics Of Perfect Crystals (MIT, Cambridge, 1975).

${ }^{17}$ S. F. Ahmad, H. Kiefte, M. J. Coulter, and M. D. Whitmore, Phys. Rev. B 26, 4239 (1982).

${ }^{18} \mathrm{~K}$. H. Brose and C. J. Eckhardt, Chem. Phys. Lett. 125, 235 (1986)

${ }^{19}$ R. C. Dye and C. J. Eckhardt, J. Chem. Phys. 90, 2090 (1989).

${ }^{20}$ E. Burgos and H. Bonadeo, Chem. Phys. Lett. 49, 475 (1977).

${ }^{21}$ G. Gafner and F. H. Herbstein, Acta Crystallogr. 17, 982 (1964).

${ }^{22}$ S. Elnahwy, M. El Hamamsy, A. C. Damask, D. E. Cox, and W. B. Daniels, J. Chem. Phys. 68, 1161 (1978). 